\title{
Hair-dye induced erythema multiforme like allergic contact dermatitis
}

\author{
Mrinal Gupta
}

Consultant Dermatologist, Treatwell Skin Centre, Jammu, India

Corresponding author: Dr. Mrinal Gupta, E-mail: drmrinalgupta@yahoo.com

\begin{abstract}
Erythema multiforme (EM) is an acute, self limited skin disease characterized by the sudden eruption of symmetrical lesions in typical targetoid fashion usually confined to face and extremities. The most commonly implicated factors include infections like herpes simplex, mycoplasma, drugs like antibiotics and anticonvulsants and vaccinations. Contact dermatitis induced EM is a rarely reported entity. Herein we present a case of a 40 year old female who presented with EM due to contact sensitivity to p-Paraphenylenediamine (PPD), a common coloring agent in hair-dyes and a potent sensitizer. Till date, there have been only a few case reports of PPD induced EM.
\end{abstract}

Key words: Allergic contact dermatitis; Erythema multiforme; Hair dye; Paraphenylenediamine; Patch test

\section{INTRODUCTION}

Erythema multiforme (EM), originally named by Hebra as erythema exudativum multiforme, is an acute self limiting mucocutaneous condition which is usually diagnosed on the basis of its characteristic clinical features like target shaped papules with a tendency for facial and acral involvement. The most common precipitating factors include viral infections and drugs [1]. EM induced by contact allergy is a rare entity. Here we report a case of EM induced by contact allergy to p-Paraphenylenediamine (PPD), a common colorant present in hair-dyes.

\section{CASE REPORT}

A 40 year old female presented in the outpatient of our department with a clinical presentation of erythema, edema and erosive dermatitis of face involving scalp margins, forehead, nape of neck, arms and hands involving both dorsal and palmar aspects. Lesions were associated with severe itching and burning sensation. Significant preauricular and cervical lymphadenopathy was also found on examination. Patient gave history of application of hair-dye 3 days prior to onset of lesions. On further probing, patient gave history of application of hair-dye twice previously about 1 year back. The first application was uneventful but the next application was followed by itching and erythema at the sites of application within 1 day. This time the patient was managed with topical potent corticosteroids and oral antihistamines. On examination the lesions were targetoid at most places with a few interspersed atypical targetoid lesions which were characteristic of EM (Fig. 1). Mucosae were not involved. No evidence of preceding Herpes infection was found and IgG and IgM for HSV 1 and 2 were also negative. No history of any drug intake prior to onset of eruption was found. These lesions resolved with application of topical potent corticosteroids with residual hyperpigmentation. As there was no precipitating factor for onset of EM, contact allergy to PPD in hair-dye was suspected to be the causative factor for EM lesions.

After the clearance of lesions, the patient was patch tested with $1 \%$ PPD in petrolatum base. The patch was applied on the back and was removed at 48 hours and was found to be $3+$ positive with erythema, induration and vesiculation which confirmed the sensitization to PPD (Fig. 2). From the history, clinical examination and patch testing, a diagnosis of contact allergy induced EM was made. 


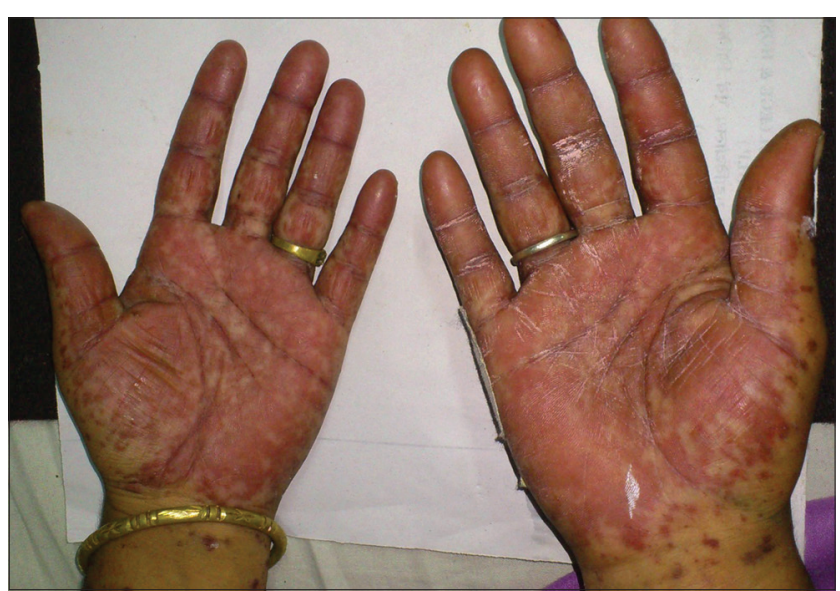

Figure 1: Erythema multiforme like lesions over the nape of neck and palms.

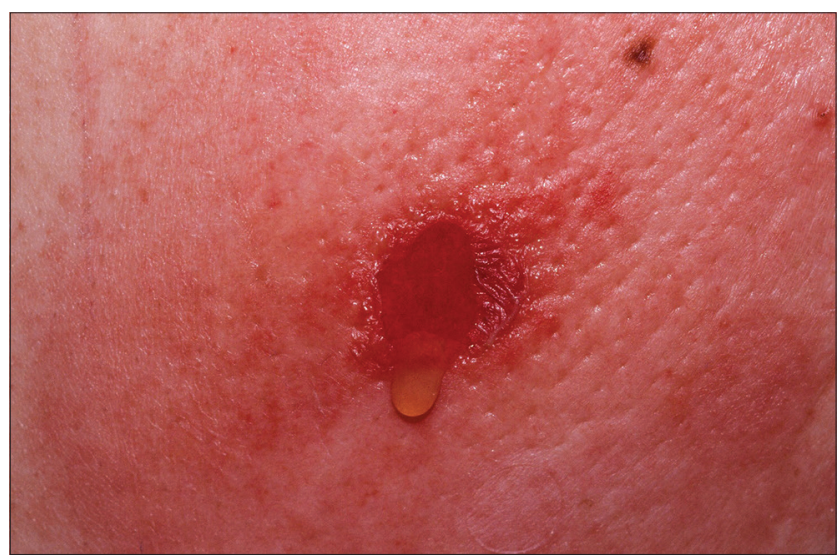

Figure 2: Patch test showing 3+ contact sensitivity to PPD.

\section{DISCUSSION}

EM is an acute self limiting condition, usually localized to face and extremities, seen mostly in young adults and characterized by characteristic target lesions. The commonest precipitating factors for EM include infections like Herpes, Mycoplasma and drugs [1]. Numerous allergens have been implicated in causing EM following contact dermatitis which includes chemicals like colophony, fragrances, epoxy resin, PPD, drugs like acetaminophen, antibiotics, metals like nickel and plant allergens like rubber and poison ivy [2] .

EM due to contact dermatitis arises usually during acute contact dermatitis or may appear after clearance of primary lesions. The pathogenic mechanism for EM following contact dermatitis is not fully known. It has been hypothesized that contact allergens may reach skin at distant sites from the site of application by travelling in peripheral mononuclear cells via the blood stream [3]. It is followed by a type III allergic reaction leading to deposition of immune complexes in the cutaneous microvasculature which leads to the cutaneous lesions. Another possible hypothesis is the type IV hypersensitivity reaction which has been proven by the presence of cytotoxic T-cells in the lesions.

PPD is a major coloring agent in hair dyes and is implicated as the most common agent causing allergic contact dermatitis to hair dyes. Along with hair dyes, PPD is also present in textile dyes, leather dyes, fur dyes, photocopiers and black rubbers. Hair dye dermatitis can present as irritant dermatitis, allergic contact dermatitis or photocontact dermatitis [4]. Other common presentations include airborne contact dermatitis, photocontact dermatitis, periorbital eczema, hand eczema, lichenoid lesions and lichen planus pigmentosus like pigmentary changes. EM like allergic contact dermatitis with hair dye is a rarely reported condition [5].

\section{CONCLUSION}

EM following hair dye dermatitis has been infrequently reported in the literature. This case was reported for the rarity of occurrence of such clinical presentation.

\section{REFERENCES}

1. Sokumbi O, Wetter DA. Clinical features, diagnosis, and treatment of erythema multiforme: a review for the practicing dermatologist. Int J Derm. 2012;51:889-902.

2. Sanchis JM, Bagan JV, Gavalda C, Murillo J, Diaz JM. Erythema multiforme: diagnosis, clinical manifestations and treatment in a retrospective study of 22 patients. J Oral Pathol Med. 2010;39:747-52.

3. Wiedemeyer K, Enk A, Jappe U. Erythema multiforme Following Allergic Contact Dermatitis; Case Report and Literature Review. Acta Derm Venereol. 2007;87:559-61.

4. Sosted H, Agner T, Andersen KE, Menne T. 55 cases of allergic reactions to hair dye: a descriptive, consumer complaint-based study. Contact Dermatitis. 2002;47:299-303.

5. Jappe U, Hausen BM, Petzoldt D. Erythema multiforme like eruption and depigmentation following allergic contact dermatitis from a paint-on henna tattoo, due to para-phenylenediamine contact hypersensitivity. Contact Dermatitis. 2001;45:249-50.

Copyright by Mrinal Gupta. This is an open access article distributed under the terms of the Creative Commons Attribution License, which permits unrestricted use, distribution, and reproduction in any medium, provided the original author and source are credited.

Source of Support: Nil, Conflict of Interest: None declared. 